\title{
Satisfacción Laboral en Trabajadores de Dos Tiendas por Departamento: Un Estudio Comparativo
}

\author{
JOB SATISFACTION AMONG EMPLOYEES FROM TWO DEPARTMENT STORES: A COMPARATIVE STUDY
}

\author{
Walter L. Arias Gallegos'1, Oscar Justo Velarde² \\ 1. Psicólogo por la Universidad Nacional de San Agustín de Arequipa, Diplomado en Gestión de Recursos Humanos, Gerencia y Supervisión en \\ Seguridad Integral, Medio Ambiente y Gerencia de Sistemas Integrados. Docente de la Universidad Católica San Pablo y director del Grupo de \\ Investigación Psyché en esta casa de estudios. E-mail: walterlizandro@hotmail.com \\ 2. Estudiante de Psicología de la Universidad Católica de Santa María. Miembro del Grupo de Investigación Psyché de la Universidad Católica San Pablo.
}

\begin{abstract}
RESUMEN
El presente trabajo se planteó como objetivo comparar los niveles de satisfacción laboral entre dos tiendas por departamento de Arequipa. Una era peruana y la otra chilena. Para ello se elaboró y validó un cuestionario de satisfacción laboral con índices aceptables de validez y confiabilidad que fue aplicado a 74 trabajadores de la empresa peruana y 148 trabajadores de la empresa chilena. Los resultados señalan que existen niveles de satisfacción más altos entre los empleados de la empresa chilena, sobre todo en cuanto al salario recibido, la organización del trabajo, las posibilidades de ascenso y la satisfacción con la empresa. En la empresa peruana se encontraron relaciones bajas, pero significativas entre el sexo del trabajador y su salario, mientras que en la empresa chilena, las relaciones más fuertes se dieron entre el salario y la organización del trabajo, la edad y la satisfacción con los subordinados y superiores.
\end{abstract}

(Arias W, Velarde 0, 2013. Satisfacción Laboral en Trabajadores de Dos Tiendas por Departamento: Un Estudio Comparativo. Cienc Trab. May-Ago; 15 [47]: 41-46).

Palabras claves: SATISFACCIÓN LABORAL, TIENDAS POR DEPARTAMENTO, CAPITAL HUMANO.

\section{ABSTRACT}

The present research settles as objective the comparison of work satisfaction levels between employees from two department stores in Arequipa. One enterprise is Peruvian and the other one is Chilean. In order to measure the satisfaction on the job, we designed and validate the Job Satisfaction Questionnaire with adequate scores of validity and reliability. This questionnaire was applied to 148 workers from Chilean enterprise and 74 workers from the Peruvian department store. The results appoint that the job satisfaction levels are higher among workers from the Chilean mall, in aspects such as payment, organization of work, promotion possibilities and satisfaction with enterprise. We also found low but significant correlations between sex and salary in Peruvian store department. Meanwhile payment and the organization of work, age and satisfaction with employees were significant in Chilean enterprise.

Key words: JOB SATISFACTION, DEPARTMENT STORE, HUMAN RESOURCES.

\section{INTRODUCCIÓN}

Se define la satisfacción laboral como un estado emocional positivo y placentero de la percepción subjetiva de las experiencias laborales del sujeto. ${ }^{1} \mathrm{Al}$ componente cognitivo de la satisfacción le denominan bienestar y es el resultado de la integración cognitiva que hacen las personas de cómo les ha ido en determinadas situaciones y contextos. Una persona tiene alto bienestar si expe-

Correspondencia / Correspondence:

Walter L. Arias Gallegos

Universidad Católica San Pablo.

Urb. Campiña Paisajista s/n Quinta Vivanco, Cercado,

Arequipa, Perú.

Fono: (51-54) 608020

e-mail:walterlizandro@hotmail.com

Recibido: 16 de Mayo de 2013 / Aceptado: 24 de Junio 2013 rimenta satisfacción con su vida y, por el contrario, una persona con bajo bienestar se siente insatisfecha. ${ }^{2}$

El bienestar psicológico tiene tres características: es subjetivo, se asocia con la vivencia de emociones positivas (más que solo la ausencia de emociones negativas) e implica hacer una valoración global de la vida. En esa misma línea, Ryff identificó seis dimensiones del bienestar psicológico: autoaceptación, autocontrol, afectos positivos, desarrollo personal, autonomía y autodeterminación. ${ }^{3}$

Pero, si bien la satisfacción laboral contribuye con el bienestar de la persona, no es temporalmente estable; y aunque la satisfacción se relaciona con las necesidades, una vez satisfechas las necesidades básicas ya no existen grandes diferencias entre las personas con respecto a esta variable. ${ }^{2}$

Los modelos teóricos de la satisfacción en el trabajo van más allá de la simple satisfacción de necesidades básicas. El modelo de Frederick Herzberg, por ejemplo, plantea que la satisfacción y la insatisfacción laboral dependen de variables diferentes entre sí. De ahí que su teoría de la satisfacción laboral se conoce como modelo bifactorial. Como la satisfacción y la insatisfacción laboral corresponden a causas diferentes, estas se constituyen en dos factores: 
los factores motivacionales intrínsecos como el progreso, el reconocimiento laboral y los logros están vinculados a la satisfacción, mientras que los factores de higiene -que son de orden extrínseco- como el salario, la política organizacional, la seguridad laboral, etc. se relacionan con la insatisfacción. ${ }^{4}$ Así pues, la idea que ha causado gran controversia es que la satisfacción y la insatisfacción no son fenómenos opuestos sino diferentes, ya que las causas de la satisfacción son diferentes de las de la insatisfacción. De este modo la ausencia de factores motivacionales no hace insatisfactorio un trabajo, como tampoco la optimización de los factores higiénicos conduce a la satisfacción laboral, porque la satisfacción y la insatisfacción laboral son fenómenos independientes. Estudios de diversa índole han señalado que la teoría de Herzberg es conceptualmente incorrecta y empíricamente inválida $a^{5}$; sin embargo, la teoría bifactorial dio pie a otros planteamientos como la teoría del enriquecimiento del trabajo.

De acuerdo con este modelo teórico, gran parte de la satisfacción y motivación que tiene un trabajador proviene de su tipo de trabajo. Este planteamiento es propio también de la teoría de la motivación basada en las características del puesto de Richard Hackman y G. R. Oldham, que proponen que las características específicas de un trabajo causan diversos estados psicológicos, los que a su vez aumentan la motivación, el rendimiento y la satisfacción con el empleo. Estos autores indicaron que la satisfacción laboral depende de ciertas características que todo puesto de trabajo debe promover: la práctica de una diversidad de habilidades, la identidad de las tareas, la importancia de la tarea, la autonomía en el trabajo y la retroalimentación. ${ }^{6}$

Tanto la teoría Herzberg como la teoría de Hackman y Oldham derivan en la implementación de medidas para que el trabajo sea más motivador. Estas medidas tienen por finalidad hacer más atractivo el trabajo y por ello forman parte de la teoría del enriquecimiento del trabajo. Entre las medidas que enriquecen el trabajo, Hackman y Oldham propusieron las siguientes: suprimir algunos controles al personal, dar más libertad y autoridad a los trabajadores, proporcionarles retroalimentación constante, ayudarles a comprender la importancia y el sentido de su trabajo como parte del proceso de producción, entre otras medidas. ${ }^{6}$ Mientras que Herzberg sugiere las siguientes alternativas para enriquecer el trabajo: plantear desafíos al trabajador, cambiar la rutina laboral, rotar al trabajador y simplificar el trabajo. ${ }^{4}$

Podemos decir que del enriquecimiento del trabajo se ha pasado al concepto de empowerment o empoderamiento, el cual consiste en darle mayores posibilidades de intervención al trabajador en las decisiones que se toman al interior de la empresa. De ahí que como estrategias de empoderamiento los trabajadores pueden participar en la formulación de objetivos empresariales ${ }^{7}$, tener presencia en los directorios o poseer cierto número acciones de la empresa en la que trabajan. ${ }^{8}$ La idea de fondo en el empoderamiento recae sobre la distribución de poder en el trabajo, de modo que este se comparte con los empleados. ${ }^{9} \mathrm{Al}$ hacer esto se estimula el sentido de pertenencia de los trabajadores en la empresa y se incrementa su compromiso porque al ser parcial o minoritariamente partícipes de las ganancias y las decisiones que se toman en el trabajo, ven la empresa como suya.

Pero no toda empresa está preparada para implementar este tipo de medidas, ya que ello depende de la cultura organizacional, el grado de horizontalidad de las jerarquías, los valores institucionales ${ }^{10} \mathrm{y}$ el nivel de cohesión institucional. ${ }^{11}$ Se ha visto también que el empoderamiento conduce al involucramiento con el trabajo, aunque el involucramiento depende de variables personales como la personalidad, y de manera muy particular, el grado de iniciativa, autoeficacia, locus de control interno, autoestima y de disfrute del trabajo; y otras de tipo laboral como la cohesión con los compañeros de trabajo, la claridad, la comodidad física y la satisfacción en el trabajo. Así pues, tres perspectivas se han usado para definir el job involvement: como variable personal, como respuesta del empleado a las condiciones de trabajo en la organización y en un sentido interactivo entre estos dos niveles. ${ }^{12}$ Hay un consenso, empero, en señalar que para las personas con alto involucramiento en el trabajo, el suyo ocupa un lugar central en sus vidas y les permite fortalecer su identidad, así como experimentar más emociones positivas como felicidad, gratitud, esperanza, perdón, engagement y flow. ${ }^{13}$

El engagement en el trabajo es definido como un estado mental positivo de realización relacionado con el trabajo, y que se caracteriza por el vigor, la dedicación y la absorción. ${ }^{14}$ Sería bueno empero, establecer diferencias entre el engagement y la satisfacción laboral, ya que si bien ambas son formas agradables de asumir el trabajo, la satisfacción laboral implica una baja activación, mientras que en el engagement la persona está altamente activada. Sin embargo, el engagement no debe confundirse con la adicción al trabajo, pues a pesar de la alta activación, esta se acompaña de sentimientos desagradables. Así pues, las personas engaged no son adictas al trabajo pues disfrutan de otras cosas en su tiempo libre y no les nace un impulso fuerte e irresistible por trabajar arduamente, porque para ellos trabajar es agradable. Además, las personas engaged son optimistas y autoeficaces, capaces de manejar los eventos que afectan sus vidas, experimentan emociones positivas y buen estado de salud. También son proactivos al movilizar sus propios recursos; por lo tanto, el engagement es un buen indicador del uso de los recursos materiales y del tiempo en el trabajo. ${ }^{15}$

El flow o estado de flujo es un constructo desarrollado por Mihaly Csikszentmihalyi ${ }^{16}$ que hace alusión a una experiencia en la que la persona se encuentra ensimismada en lo que está haciendo porque disfruta de dicha actividad, a tal punto que pierde la noción del tiempo. Este tipo de experiencias son comunes, por ejemplo, entre los trabajadores que tienen mayor nivel de compromiso, involucramiento, motivación y satisfacción en o por su trabajo. El locus de control interno también se ha asociado con un mayor nivel de involucramiento en las actividades laborales, así como diversas variables como los años de tiempo de servicio ${ }^{17}$, la experiencia laboral, la capacidad intelectual, el nivel ocupacional $^{18}$, y las expectativas de los jefes respecto del rendimiento de los trabajadores. ${ }^{19,20}$ En ese sentido hay estudios que señalan que ser promovido se relaciona con factores motivacionales tanto antes como después del ascenso. Es decir, que los trabajadores más activos y motivados son los que tienen mayor probabilidad de progresar en el ámbito laboral, y además aquellos que han cambiado de estatus se esfuerzan más en el trabajo y desarrollan más conductas de innovación. ${ }^{21}$

En relación con los conceptos analizados previamente, podemos concluir que la satisfacción laboral se entiende bidireccionalmente, es decir, como causa y como efecto. En el primer caso, la satisfacción laboral afecta la motivación del trabajador. De hecho, entre satisfacción laboral y motivación existen relaciones muy estrechas, como hemos podido apreciar ${ }^{18}$, ya que un trabajador satisfecho está motivado para trabajar y se involucra más con su trabajo. ${ }^{17}$

Entre los motivadores que tienen mayor influencia en el trabajo de los empleados se tienen el salario (en la medida que permite 
cubrir las necesidades básicas), el logro de las metas, el reconocimiento y la equidad en el trabajo. ${ }^{22}$ Precisamente este último factor, el de la equidad, apunta a las relaciones humanas que se conforman en el trabajo. Las relaciones con los compañeros de trabajo y los superiores pueden tener efectos a largo plazo en la satisfacción del trabajador. ${ }^{23}$ De hecho, las relaciones de apoyo entre compañeros, la retroalimentación sobre el desempeño y el desarrollo de competencias de los empleados facilitan el engagement ${ }^{15} \mathrm{y}$, por ende, hacen trabajadores más satisfechos.

Sin embargo, así como la satisfacción laboral influye en la productividad, el ausentismo y la rotación de los trabajadores ${ }^{24}$, también puede verse influida por diversos factores. De ahí que la satisfacción laboral es una variable multicausal y multifactorial. En algunos casos, suele verse afectada por situaciones que implican una ruptura del contrato psicológico. ${ }^{25}$ Por ejemplo, cuando el trabajador percibe que no se cumplen los ofrecimientos que se le hacen al acceder a un trabajo se produce una ruptura entre empleador y empleado que afecta la satisfacción laboral del segundo. ${ }^{26}$ Entre las variables organizacionales que afectan la satisfacción laboral se tienen el verticalismo, la estructura organizacional, clima organizacional negativo, etc. ${ }^{27}$

Según algunas estimaciones, el año 2000 el 50,7 por ciento de los trabajadores en Estados Unidos se sentía insatisfecho con su trabajo. ${ }^{24}$ En el Perú, dadas las condiciones de inestabilidad laboral, subempleo de los trabajadores, bajos salarios, inseguridad, autoritarismo de los empleadores, etc., la satisfacción laboral se encuentra seriamente mellada. Sin embargo, el panorama futuro es promisorio ya que la inversión privada ha aumentado a un ritmo vertiginoso y se han desarrollado mejoras sustantivas a la legislación laboral, lo que supone que en los años venideros las condiciones laborales se alinearán con las últimas tendencias y hallazgos en materia de desarrollo organizacional. Ahora bien, uno de los países que más ha invertido en Perú ha sido Chile. Particularmente en Arequipa -ciudad en que tiene lugar el presente estudio-, donde en los últimos tres años el sector comercial ha evidenciado un importante crecimiento debido a la construcción de malles y centros comerciales con capitales de origen chileno. El presente estudio pretende comparar los niveles de satisfacción laboral en los empleados de dos tiendas por departamentos de Arequipa (una nacional y otra chilena) en función del salario que reciben, las relaciones entre compañeros de trabajo de diferentes jerarquías, las posibilidades de promoción y la organización del trabajo en la empresa. De ello se desprende que la pregunta de investigación que orienta este estudio es:

¿Cuál será el nivel de satisfacción laboral de los empleados que trabajan en dos tiendas por departamentos de Arequipa, siendo una peruana y la otra chilena?

\section{MATERIAL Y MÉTODOS}

\section{Muestra}

La muestra está constituida por 222 trabajadores de dos tiendas por departamento de Arequipa. En un primer caso, se extrajo una muestra de 148 trabajadores de una empresa chilena que cuenta con 1.528 trabajadores, mientras que para el caso de la empresa peruana se tomó una muestra de 74 trabajadores de una población de 356. Los trabajadores realizaban labores de venta, caja, limpieza, seguridad y atención al cliente. La selección de la muestra se realizó mediante la técnica de muestreo por cuotas. Las muestras se obtuvieron con un nivel de significancia de 99\% y un error de estimación de $1 \%(p<0.01)$.

\section{Instrumentos}

Como instrumento de investigación se diseñó y elaboró un cuestionario que recoge información relativa a la satisfacción laboral a partir del salario recibido, el tipo de tarea que se realiza, las relaciones interpersonales, las posibilidades de promoción y la organización.

El cuestionario cuenta con una estructura de escala tipo Likert y ocho preguntas con un rango de respuesta que va desde muy satisfecho hasta nada satisfecho.

Tabla 1.

Preguntas del Cuestionario de satisfacción laboral.

1. ¿Está usted satisfecho actualmente con su salario?

2. ¿Está satisfecho con el tipo de trabajo que usted hace?

3. ¿Con los empleados que dependen de usted?

4. ¿Está usted satisfecho con los jefes y superiores?

5. ¿Está usted satisfecho con los compañeros de trabajo?

6. ¿Se siente satisfecho con sus posibilidades de ascenso?

7. ¿Con la organización del trabajo que actualmente hace?

8. ¿Qué satisfacción tiene usted en su empresa?

Para efectos de la presente investigación, se validó el cuestionario y se obtuvo su índice de confiabilidad. Estos datos se presentan en la sección de resultados. También se recogieron datos tales como sexo, tiempo de servicios, estado civil, edad y ocupación.

\section{Procedimiento}

Se solicitó los permisos para evaluar a los trabajadores que laboran en las empresas que formaron parte de la muestra. Este permiso fue concedido solo si se mantenía en reserva el nombre de la empresa de la que proceden las personas que fueron evaluadas. La recolección de la información se hizo a través del cuestionario de satisfacción laboral, que fue aplicado a los trabajadores en su horario de trabajo en ambientes privados que fueron concedidos en cada caso.

\section{RESULTADOS}

\section{Validez y confiabilidad del instrumento}

En la Tabla 2 se aprecian los valores de las correlaciones item-test que se procesaron para obtener la validez de criterio del cuestionario utilizado. Todas las correlaciones fueron superiores a 0,20 (mínimo $=0,443$, ítem 5 y máximo $=0,857$, item 8 ).

Tabla 2.

Correlaciones ítem-test del instrumento.

$\begin{array}{llllllllll} & 1 & 2 & 3 & 4 & 5 & 6 & 7 & 8 & T \\ 1 & 1 & 0,49 & 0,38 & 0,27 & 0,16 & 0,35 & 0,47 & 0,57 & 0,72 \\ 2 & & 1 & 0,35 & 0,34 & 0,21 & 0,31 & 0,43 & 0,56 & 0,69 \\ 3 & & & 1 & 0,36 & 0,16 & 0,28 & 0,34 & 0,52 & 0,68 \\ 4 & & & & 1 & 0,25 & 0,11 & 0,27 & 0,47 & 0,59 \\ 5 & & & & & 1 & 0,09 & 0,09 & 0,34 & 0,44 \\ 6 & & & & & & 1 & 0,26 & 0,44 & 0,54 \\ 7 & & & & & & & 1 & 0,58 & 0,67 \\ 8 & & & & & & & & 1 & 0,85 \\ \mathrm{~T} & & & & & & & & & 1 \\ \mathrm{p}<0,01 & & & & & & & & & \end{array}$


Para obtener la validez de constructo se procedió en primer lugar a obtener el valor KM0, que resultó ser 0,850; por lo tanto, es factible realizar un análisis factorial. Se procedió entonces a hacer un AFE (Análisis Factorial Exploratorio) con rotación varimax en el programa SPSS 19.0, teniendo como resultado la existencia de dos factores, como se aprecia en la Tabla 3.

El primer factor contiene los items 1 (0,763), 2 (0,667), 6 (0,661), 7 $(0,722)$ y $8(0,759)$ con sus respectivas cargas factoriales. Mientras que el factor 2 contiene los items 3, 4 y 5 con las siguientes cargas factoriales: 0,341, 0,690 y 0,818. Dados los contenidos de los ítems que componen cada factor, el primer factor fue denominado: condiciones de trabajo, y el segundo factor: relaciones humanas. Cada uno cuenta con valores de consistencia interna aceptables ( $\alpha$ $=0,880$ para el primer factor y $\alpha$ $=0,475$ para el segundo factor). Asimismo, se determinó la confiabilidad mediante el coeficiente Alpha de Cronbach, obteniendo un valor de 0,759 , lo que nos lleva a concluir que el instrumento utilizado es válido y confiable.

\section{Valores descriptivos y correlaciones}

Con respecto a los valores descriptivos tenemos que la muestra de la empresa peruana está conformada por 29 varones (39,2\%) y 45 mujeres (60,8\%). Asimismo, la media del tiempo de trabajo es de ocho meses con un tiempo mínimo de un mes y un tiempo máximo de 74 meses, es decir, seis años aproximadamente. Con respecto al estado civil $84,6 \%$ son casados, $13,8 \%$ son solteros y $1,5 \%$ son divorciados. La edad promedio fue de 24 años con una desviación estándar de $\pm 4,9$, y un valor mínimo de 18 años y un máximo de 38 años. En cuanto a la ocupación, el 76,1\% se dedica a las ventas, un 8,7\% a despachar en caja, 8,7\% a atender al cliente, el 4,3\% a limpieza y el 2,2\% a funciones de seguridad.

Los valores descriptivos acerca de la satisfacción laboral por ítem se aprecian en la Tabla 4, notándose medias bajas para los trabajadores de la empresa peruana en consideración del rango de respuestas.

Tabla 4.

Descriptivos de satisfacción laboral en la empresa peruana.

$\begin{array}{lcccccccc} & \mathbf{1} & \mathbf{2} & \mathbf{3} & \mathbf{4} & \mathbf{5} & \mathbf{6} & \mathbf{7} & \mathbf{8} \\ \text { Media } & 1,45 & 1,60 & 1,09 & 1,60 & 1,95 & 1,62 & 1,54 & 1,64 \\ \text { Desv Stand. } & 0,95 & 0,75 & 1,11 & 1,01 & 0,89 & 0,80 & 0,92 & 0,81 \\ \text { Varianza } & 0,90 & 0,57 & 1,23 & 1,03 & 0,80 & 0,64 & 0,85 & 0,66 \\ \text { Asimetría } & -0,76 & -0,57 & 0,36 & -0,18 & -0,61 & -0,48 & -0,22 & -0,49 \\ \text { Kurtosis } & -0,91 & 0,04 & -1,37 & -1,04 & -0,26 & -0,15 & -0,76 & -0,15 \\ \text { Rango } & 3 & 3 & 3 & 3 & 3 & 3 & 3 & 3 \\ \text { Mínimo } & 0 & 0 & 0 & 0 & 0 & 0 & 0 & 0 \\ \text { Máximo } & 3 & 3 & 3 & 3 & 3 & 3 & 3 & 3\end{array}$

También se obtuvieron correlaciones entre la edad, el sexo, el estado civil, el tiempo de servicios y la ocupación con las respuestas de cada ítem. Los datos se observan en la Tabla 5. El único valor significativo fue el de la correlación entre sexo y salario $(r=-0,24)$ lo que sugiere que los varones se encuentran más satisfechos con el sueldo que reciben.
Tabla 5.

Correlaciones entre la satisfacción laboral y las variables sociolaborales en los trabajadores de la empresa peruana.

$\begin{array}{lcccccccc} & \mathbf{1} & \mathbf{2} & \mathbf{3} & \mathbf{4} & \mathbf{5} & \mathbf{6} & \mathbf{7} & \mathbf{8} \\ \text { Edad } & -0,08 & 0,07 & 0,09 & -0,05 & 0,01 & -0,13 & 0,08 & 0,08 \\ \text { Sexo } & -0,24^{*} & 0,03 & -0,21 & 0,06 & 0,06 & -0,06 & -0,17 & -0,08 \\ \text { Tiempo } & 0,01 & 0,07 & 0,03 & 0,04 & 0,12 & -0,19 & 0,05 & 0,15 \\ \text { Est, civil } & 0,04 & 0,04 & 0,04 & -0,14 & -0,06 & 0,06 & 0,10 & 0,06 \\ \text { Ocupac, } & -0,12 & 0,03 & 0,02 & 0,12 & -0,10 & 0,10 & 0,16 & -0,16\end{array}$

Tabla 6.

Descriptivos de satisfacción laboral en la empresa chilena.

$\begin{array}{lcccccccc} & \mathbf{1} & \mathbf{2} & \mathbf{3} & \mathbf{4} & \mathbf{5} & \mathbf{6} & \mathbf{7} & \mathbf{8} \\ \text { Media } & 2,53 & 2,21 & 2,00 & 2,08 & 2,17 & 2,16 & 2,29 & 2,27 \\ \text { Desv Stand. } & \mathbf{0 , 6 3} & 0,55 & 0,79 & 0,70 & 0,80 & 0,75 & 0,76 & 0,59 \\ \text { Varianza } & 0,40 & 0,30 & 0,63 & 0,49 & 0,64 & 0,57 & 0,57 & 0,35 \\ \text { Asimetría } & -1,35 & -0,19 & -0,42 & -0,70 & -0,63 & -0,85 & -1,03 & -0,56 \\ \text { Kurtosis } & 2,18 & 1,09 & -0,33 & 1,03 & -0,29 & 0,86 & 0,96 & 1,60 \\ \text { Rango } & 3 & 3 & 3 & 3 & 3 & 3 & 3 & 3 \\ \text { Mínimo } & 0 & 0 & 0 & 0 & 0 & 0 & 0 & 0 \\ \text { Máximo } & 3 & 3 & 3 & 3 & 3 & 3 & 3 & 3\end{array}$

Tabla 7.

Correlaciones entre la satisfacción laboral y las variables sociolaborales en los trabajadores de la empresa chilena.

$\begin{array}{lcccccccc} & \mathbf{1} & \mathbf{2} & \mathbf{3} & \mathbf{4} & \mathbf{5} & \mathbf{6} & \mathbf{7} & \mathbf{8} \\ \text { Edad } & 0,05 & 0,02 & 0,16^{*} & 0,19^{*} & 0,11 & 0,04 & 0,05 & 0,05 \\ \text { Sexo } & -0,15 & -0,08 & -0,02 & -0,00 & -0,03 & -0,12 & -0,13 & -0,13 \\ \text { Tiem } & 0,17^{*} & 0,08 & -0,10 & -0,03 & -0,02 & -0,07 & 0,25^{*} & 0,08 \\ \text { Ecivil } & -0,08 & 0,11 & -0,02 & -0,11 & -0,11 & 0,05 & 0,00 & -0,04 \\ \text { Ocup } & -0,11 & -0,10 & 0,02 & 0,04 & 0,07 & -0,07 & 0,00 & 0,02 \\ p<0,01 & & & & & & & & \end{array}$

Con respecto a la empresa chilena se tiene que la muestra está compuesta por 86 varones $(58,1 \%)$ y 61 mujeres $(41,9 \%)$. La media del tiempo de trabajo fue de 21 meses con un tiempo mínimo de un mes y un tiempo máximo de 72 meses, es decir, al igual que en la empresa peruana. En cuanto al estado civil, el 70,3\% está casado y el $29 \%$ está soltero, mientras que el 0,7\% está divorciado. La edad promedio fue de 25 años con una desviación estándar de $\pm 4,1$, y un valor mínimo de 19 años y un máximo de 43 años. En cuanto a la ocupación, el 58,7\% se dedica a las ventas, 19,6\% a atender al cliente, $12,2 \%$ a despachar en caja, el 6,1\% a la limpieza y el 3,4\% a las funciones de seguridad.

En la Tabla 6 se pueden observar los valores descriptivos acerca de la satisfacción laboral por ítem para los trabajadores de la empresa chilena. Estos valores son, a simple vista, superiores a los de la empresa peruana.

Las correlaciones entre la edad, el sexo, el estado civil, el tiempo de servicios y la ocupación con las respuestas de cada item se detallan en la Tabla 7. Los valores más significativos fueron entre el tiempo de trabajo y el salario recibido $(\mathrm{r}=0,17)$, entre el tiempo de trabajo y la organización del mismo $(r=0,25)$, así como entre la edad y la satisfacción con los subordinados $(r=0,16)$ y superiores $(r=0,19)$. Se observa, en consecuencia, que hay más relaciones entre las variables estudiadas en los trabajadores de la empresa chilena.

\section{Valores comparativos}

Finalmente, se procesaron los valores obtenidos por item, mediante la prueba $t$ student para determinar si las diferencias 
que se aprecian entre los trabajadores de ambas muestras son significativas.

En la Tabla 8 se puede ver que todos los valores de la t obtenida son superiores a los de la t crítica, por tanto, las diferencias entre ambas muestras son significativas en todos los items. Pero de manera muy particular en las cuestiones que tienen que ver con el salario recibido (ítem 1), el tipo de trabajo que se realiza (item 2), las posibilidades de ascenso (ítem 6), la organización del trabajo (item 7) y la satisfacción con la empresa (item 8).

Tabla 8.

Comparación de valores mediante la t student.

\begin{tabular}{|lcc|c|c|}
\hline İtem & $\mathrm{t}$ crítica & $\mathrm{t}$ obtenida & $\mathrm{gL}$ & $\mathrm{p}$ \\
\hline 1 & 2,345 & 72,437 & & \\
\hline 2 & 2,345 & 47,506 & & \\
\hline 3 & 2,345 & 49,002 & & \\
\hline 4 & 2,345 & 28,226 & 220 & 0,01 \\
\hline 5 & 2,345 & 11,334 & & \\
6 & 2,345 & 34,324 & & \\
7 & 2,345 & 39,193 & & \\
8 & 2,345 & 45,652 & & \\
\hline
\end{tabular}

En cambio, aunque significativas, en menor grado se tienen las diferencias con respecto a las relaciones con los jefes (item 4) y los compañeros (item 5).

\section{DISCUSION}

La satisfacción laboral ha sido uno de los constructos más estudiados dentro de la psicología organizacional, pues afecta directamente la moral del trabajador. Aunque una primera aproximación de la psicología hacia el trabajo ha tenido que ver con la aplicación de los principios del análisis funcional de la conducta (AFC) a la productividad ${ }^{28}$, hoy son más relevantes la satisfacción y la motivación del trabajador, por cuanto la productividad y otras variables que afectan la rentabilidad de la empresa ${ }^{29}$ dependen de la satisfacción y las emociones positivas que se experimentan en el trabajo. De hecho, estas variables tienen efectos en el significado que el trabajo tiene para el trabajador, por ello poseen el potencial para afectar su desempeño.

Así pues, el significado del trabajo, como un conjunto de creencias, actitudes y valores hacia el trabajo, si bien se aprenden en la familia, puede cambiar bajo la influencia de la experiencia laboral. ${ }^{30}$ Una empresa con un pésimo clima organizacional, con una estructura vertical y con escasa preocupación por las necesidades del trabajador, está condenada a fracasar como negocio.

En el presente trabajo de investigación se han comparado los niveles de satisfacción laboral entre los trabajadores de dos tiendas por departamento de Arequipa. Una de ellas peruana y otra chilena. Para ello se ha elaborado y validado un cuestionario que valora la satisfacción laboral mediante ocho preguntas con cuatro niveles de respuesta en una escala de Likert.

Los resultados señalan que los niveles de satisfacción laboral son superiores en los trabajadores de la empresa chilena, sobre todo en los aspectos relativos al salario, el tipo de trabajo realizado, las posibilidades de ascenso, la organización del trabajo y la satisfacción con la empresa. Esto significa que los trabajadores de la tienda por departamentos de Chile reciben un mayor sueldo, perciben su trabajo como más agradable y organizado y se sienten más satisfechos con la empresa para la que trabajan. Estas cuestiones laborales han de afectar la integración de los trabajadores a la organización y su grado de compromiso para con la misma. El compromiso organizacional hace alusión al deseo de realizar elevados esfuerzos por el bien de la institución, así como al anhelo de permanecer en la organización y aceptar sus principales objetivos y valores. ${ }^{31}$ En ese sentido, si los trabajadores perciben que la empresa les otorga ciertos beneficios como un sueldo que les permita satisfacer sus necesidades y desplegar sus potencialidades en el trabajo, se producirá un sentimiento que les impelerá a actuar valorando la organización de manera positiva y a movilizar sus recursos emocionales hacia la consecución de las metas organizacionales.

Por esta razón, la integración de las personas a la organización es un asunto de vital importancia, ya que tiene efectos en la conducta del trabajador, la aceptación de las metas, los valores y la cultura de la organización, disminuyendo el ausentismo y la rotación de personal. En consecuencia, en la medida que se asuman los intereses y objetivos de la empresa, se desarrollará un mejor sentido del trabajo. Es conveniente, entonces, poner atención a la satisfacción laboral de los trabajadores.

En esta investigación se encontró también que en los trabajadores de la empresa chilena aquellos que llevan más tiempo trabajando reciben un mejor salario y se sienten más satisfechos con la organización de sus tareas laborales. Ello supone, aunado al alto nivel de satisfacción con respecto a la promoción de los trabajadores, que se implementan medidas adecuadas de ascensión de puestos y de organización del trabajo. Nótese, por ejemplo, que en la empresa chilena hay mayor cantidad de trabajadores, cuya función es atender al cliente (8,7\% en la empresa peruana y 19,6\% en la empresa chilena).

Asimismo, a mayor edad de los trabajadores más satisfactoria es la relación con sus compañeros de trabajo y sus subordinados; posiblemente porque con el paso del tiempo disminuye la competencia entre los trabajadores. Por el lado de la empresa peruana, la única relación significativa se dio entre el sexo y el salario, lo que es un indicador de que los varones ganan más que las mujeres $y$, por ende, que podrian existir prácticas sexistas en esta empresa o la organización del trabajo se basa de manera diferencial en el sexo del trabajador.

Cabe mencionar, empero, que si bien las relaciones mencionadas son significativas, la fuerza de las relaciones halladas es baja. Con respecto a la empresa de Perú, dado que se hallaron diferencias menos significativas en la satisfacción que deviene de las relaciones con jefes y compañeros, podemos colegir que en ambas empresas las relaciones que se establecen con los compañeros de trabajo cumplen una función amortiguadora sobre los aspectos que son percibidos negativa o potencialmente perjudiciales en el trabajo.

De esta manera, las relaciones humanas en el trabajo constituyen una vía para afrontar el estrés laboral y la satisfacción laboral, ya que la confianza en las relaciones interpersonales tiene gran significancia en la satisfacción laboral. Aquí tienen un peso importante las relaciones con los superiores, pues el liderazgo también tiene un impacto positivo en la satisfacción laboral. ${ }^{32}$ En conclusión, la confianza es una ventaja competitiva ${ }^{14}$ y un facilitador de las relaciones humanas y las actitudes positivas en el trabajo, pero, lamentablemente, los datos de diversos estudios señalan que dos tercios de trabajadores tienen poca confianza en sus empleadores. ${ }^{32}$ 
Por otro lado, las diferencias halladas en las dos tiendas por departamento que formaron parte de este estudio nos permiten concluir que la empresa chilena favorece el desenvolvimiento de sus trabajadores al propiciar mayores niveles de satisfacción laboral, porque aparentemente se preocupa por el diseño de la tarea, el ambiente de trabajo, las necesidades materiales de los trabajadores, así como su estructura organizacional y las relaciones humanas entre los empleados. ${ }^{14}$ En ese sentido, la satisfacción de un empleado no debe depender solamente de los incentivos, ya que además de tener un efecto limitado, constituyen un peligro potencial porque centran su atención en los objetivos y preocupaciones individuales, cuando de lo que se trata es que el trabajador se comprometa con los objetivos de la organización y los asuma de manera evidente con su trabajo. ${ }^{33}$
Es muy probable, entonces, que estas empresas difieran en los recursos que invierten en la salud y seguridad del trabajador, en su capacitación y comodidad, así como en la organización eficiente del trabajo y el grado de control de los procesos de atención al cliente. Pensamos que las diferencias encontradas se deben, ante todo, a que las empresas chilenas funcionan inmersas en una cultura empresarial que ha sido característica del vecino país del sur desde hace muchos años, mientras que en el Perú no se han difundido lo suficiente los enfoques centrados en el trabajador -según los cuales la eficiencia de la producción depende directamente de la satisfacción del trabajador- sino que prima una mentalidad que enfatiza la producción a costa de la satisfacción laboral.

Deseamos agradecer, para finalizar, a los trabajadores y directivos de ambas empresas por su gentil colaboración.

\section{REFERENCES}

1. Fonseca Y. Estudio de la satisfacción laboral en los trabajadores de la empresa comercializadora y distribuidora de medicamentos (EMCOMED) de la provincia de Granma. Rev Cubana Salud Trabajo. 2010;11(2):15-19.

2. Casullo MM, Castro A. Evaluación de bienestar psicológico en estudiantes adolescentes argentinos. Rev psicol (Lima). 2000;18(1):35-68.

3. Contini N, Coronel P, Levin M. Estevez A. Estrategias de afrontamiento y bienestar psicológico en adolescentes escolarizados de Tucumán. Rev Psicol (Lima). 2003; 21(1):179-199.

4. Herzberg F. Una vez más: ¿Cómo se motiva a los empleados? En: Harvard Business Review. Barcelona: Deusto; 2004. p.53-80.

5. León F, Sepúlveda MV. Satisfacciones e insatisfacciones herzbergianas en el trabajo, Rev Latinoam Psicol. 1979;11(1):93-113.

6. Schutz D. Psicologia industrial. México: McGraw-Hill; 1998.

7. Levinson $\mathrm{H}$. ¿Dirección de los objetivos de quién? En: Harvard Business Review. Barcelona: Deusto; 2004. p. 81-110.

8. Chiavenato I. Gestión del talento humano. McGraw-Hill: México; 2009.

9. Meliá JL, Oliver A, Tomás JM. El poder en las organizaciones y su medición. El cuestionario de poder formal e informal, Rev Latinoam Psicol. 1993;25(2):139-155.

10. Lacouture G, Factores motivacionales del apoderamiento (enpowerment) en la organización. Rev Latinoam Psicol. 1996;28(1):135-147.

11. Reidl $L$, Reyes $L$, Sanguinetti $Y$. Un modelo aplicado al estudio de la cohesión institucional. Rev Latinoam Psicol. 1980;12(1):95-108.

12. Martínez-López ME. Variables asociadas a la involucración en el trabajo. Rev Latinoam Psicol. 1990;22(2):211-222.

13. Salanova M, Martínez I, Llorens S. Psicología organizacional positiva. En: Palaci JF, coordinador. Psicología de la organización. Madrid: Pearson Education; 2005. p.349-376.

14. Acosta $H$, Salanova M, Llorens $S$. ¿Cómo predicen las prácticas organizacionales el engagement en el trabajo en equipo?: El rol de la confianza organizacional. Cienc Trab. 2011;13(41):125-134.

15. Bakker A, Demerouti $E$, Xanthopoulou S. ¿Cómo los empleados mantienen su engagement en el trabajo? Cienc Trab. 2011;13(41):135-142.

16. Csikszentmihalyi M. Fluir en los negocios Barcelona: Kairó; 2003.

17. Martínez-Lugo M. La involucración con el trabajo y su relación con la edad, el locus de control y los años de servicio. Rev Latinoam Psicol. 1988;20(2):137-148.
18. Gómez A, Benítez C, Guillén c, Gala FJ, Lupiani M. Motivación y satisfacción laboral. En: Guillén $C$, Guil $R$, coordinadores. Psicología del trabajo para relaciones laborales. Madrid: McGraw-Hill; 2000. p.195-209.

19. Livingston S. El Pigmalión en la dirección empresarial. En: Harvard Business Review. Barcelona: Deusto; 2004. p.185-211.

20. León F. Consideración del supervisor y consecuencias de rendimiento anticipadas por el subordinado. Rev. Latinoam Psicol. 1978;10(2):141-163.

21. Ripoll P, Caballer A, Martínez V, García E, Peiró JM. Antecedentes y consecuencias del cambio de nivel jerárquico en el trabajo. Rev Latinoam Psicol. 1988;30(2):203-222.

22. Baron RA. Psicología. McGraw Hill: México; 1997.

23. Palaci FJ. Socialización y resocialización en las organizaciones. En: Palaci FJ, coordinador. Psicologia de la Organización. Madrid: Pearson Education; 2005. p.73-100.

24. Robbins, SP. Comportamiento organizacional. México: Prentice Hall; 1999.

25. Topa G. El contrato psicológico: Un nuevo marco para comprender las relaciones dentro de las organizaciones. En: Palaci FJ, coordinador. Psicologia de la Organización. Madrid: Pearson Education; 2005. p.101-119.

26. Topa $G$, Morales F. Identificación organizacional y proactividad personal en grupos de trabajo: Un modelo de ecuaciones estructurales. Anales psicol. 2006;22(2):234-215.

27. Alcover CM. Cultura y clima organizacional. En: Gil F, Alcover CM, Coordinadores. Introducción a la Psicología de las Organizaciones. Madrid: Alianza Editorial; 2003. p.387-414.

28. Jiménez A. El análisis conductual aplicado como técnica para aumentar la productividad. Rev Latinoam Psicol. 1978;10(1):17-23.

29. Ficshman D. La alta rentabilidad de la felicidad. Lima: UPC; 2011.

30. Gracia FJ, Martín P, Rodríguez I, Peiró JM. Cambios en los componentes del significado del trabajo durante los primeros años de empleo: Un análisis longitudinal. Alanes psicol. 2001;17(2):201-217.

31. Betanzos N, Paz F. Análisis psicométrico del compromiso organizacional como variable actitudinal. Alanes psicol. 2007;23(2):207-215.

32. Yañez $R$, Arenas $M$, Ripoll M. El impacto de las relaciones interpersonales en la satisfacción laboral general. Liberabit. 2010;16(2):193-202.

33. Topa $G$, Morales F. Identificación organizacional y proactividad personal en grupos de trabajo: Un modelo de ecuaciones estructurales. An psicol. 2006;22(2):234-242. 